\title{
Research on dynamic viewport display method for automatic generation of power flow diagram
}

\author{
Wenchong Fang ${ }^{1}$,Taifeng Kang ${ }^{2}$ a ${ }^{*}$, Bin Zhang ${ }^{2}$, Shouyu Liang ${ }^{1}$, Ruipeng \\ Zhang $^{1}$, Fengqing Zhao ${ }^{2}$, Yonggang $\mathrm{Li}^{2}$ \\ ${ }^{1}$ China Southern Power Grid (CSG) dispatching and control center, Guangzhou, China \\ ${ }^{2}$ Beijing Sifang Automation CO., LTD., Beijing, China \\ aarfa2011_1@163.com \\ * Corresponding author
}

Keywords: power flow diagram; panorama dispatching; diagram automatic generation; geographic information; smart grid

\begin{abstract}
Power flow diagram is an important tool of modern Energy Management System (EMS) for system operators to schedule the operation and maintenance plan, examine security situation and dispatch in real-time. Manually creation and maintenance of power flow diagram of large scale power grid is time consuming and error prone. In the previous research, exposure of the detail information inside substations that is of interest to system operators and incremental update of the generated diagrams are not supported for diagram automatic generation of power flow diagrams. In this paper, we propose dynamic viewport display method for automatic generation of large scale power flow diagram. Firstly, integrative management of model and graph of the power grid is introduced. Then dynamic viewport display method is developed to automatically generate the power flow diagram for large scale power grid. With our proposed approach, essential layout inside the substation is exposed to support systematic simulation and control. Besides, incremental mapping is supported to increase the efficiency for diagram generation and maintenance. The method could not only improve efficiency and practicability of power flow diagram automatic generation, but also provide the systematic and integrative map for simulation and control of the power grid. The example in actual projects has shown its advantages.
\end{abstract}

\section{Introduction}

Power flow diagram provides the overview of systematic outline and topology, real-time electrical information of circuit breakers and power flow, which is an important tool of modern automation system of power grid for system operators to schedule the operation and maintenance plan, check security situation and dispatch in real-time of the grid. In tradition, such diagrams are manually created and maintained by regions/substations due to the scale of power grid and large amount of workload. However, such region/substation based power flow diagrams provide less convenience than the diagrams of the overall system, as the selection of the regions is subjective and the view of diagrams is divided, especially for the boundary between regions. Besides, model and graph resource are allocated into separate diagrams, which increases the difficulty to integrative search and fast locate the equipments on the diagram.

As manually creation and maintenance of power flow diagram of large scale power transmission network is very time consuming and error prone, automatic generation of such diagrams have draw more and more attentions ${ }^{[1,2,3,4,5,6]}$. There are some general requirements for such technologies, such as the clearance and elegance of the layout and sketch in appropriate display areas, less crossings, real-time measurement automatically mapped as well as high display efficiency, etc. References [3, 4] researched on single-line diagram generation for power grid and proposed method for automatic layout of elements in substation. References [5,6] researched on diagram generation method for power grid based on geographic information. These methods for single-line diagram automatic generation are limited to single substations without systematic vision of the power grid and the 
methods based on geographic information provide with no exposure of bus bars in substation, which limits the utilities integrated with advanced applications for simulation, dispatching and remote control. Besides, integrative management and update of model and graph, incremental update of the generated and efficiently display and operate on the generated diagrams have not been mentioned or implemented which may affect the feasibility and applicability of diagram automatic generation.

In this paper, we research on diagram automatic generation solution and propose dynamic viewport display method for automatic generation of power flow diagram for large scale of power grid. In the proposed method, firstly, integrative management of model and graph of the power grid is introduced. Then dynamic viewport display method is developed to automatically generate the power flow diagram for mega power grid. With the proposed approach, the elements of the diagram are displayed on an elegant line and column based layout and essential layout of bus bars is exposed for systematic simulation or dispatch. Only the carefully calculated elements within the viewport are displayed and typical browsing operations are also supported efficiently. The method could not only improve efficiency of display and operation on power flow diagram, but also provide the top and graceful vision for the whole of power grid.

\section{Diagram automatic generation for power grid}

Smart grid and diagram automatic generation. Traditional power grid is a simple, rigorous and one-way system, and there are limited ways for integrative control of generation, power transmission and power consuming. Therefore, little flexibility and self-healing ability is available for such system which is completely dependent on the physical redundancy of system components. Information of traditional power grid is separated into multiple islands and barely utilized to improve the efficiency and economics of the system.

Compared with traditional power grid, smart grid is much stronger and more reliable, flexible and interactive. It integrates all kinds of real time operation and management information to provide analysis, diagnosis and optimization strategies for system operation and management. More comprehensive and decision supporting information is provided to system schedulers and operators to enhance reliability, efficiency, economics and sustainability of the power grid.

As the toolkits for presenting system operation information and real-time status interactively and intuitively, GUI (graphical user interfaces) is one of the key components for smart dispatching to enhance the ability of situation awareness and perform system management and real-time control. Automatic generation of systematic diagrams is one of the key technologies for smart grid.

Types and challenges of automatic generated diagrams. According to the information utilized, the style and the usage of the generated diagram, there are different types of automatically generated diagrams, including single-line substation diagram, geographic information based power flow diagram and one-line formatted power flow diagram.

Single-line substation diagram is the layout of the equipments in the substation, including bus bar, transformer, circuit breaker, load, etc. It satisfies the requirement for information investigation and system operation in substation level; however, all the information is limited in substation level, and it is impossible to provide system wide information.

Geographic information based power flow diagram is the layout of the power flow diagram according to the geographic information with substations, lines, etc. It could provide intuitive and systematic view. However, no detail of substation is provided, and it is unsuitable for direct operation and dispatch of the power grid.

One-line formatted Power flow diagram is the layout of the power flow diagram in one-line format with substations, lines, etc. It can provide systematic view of the whole power grid and it is suitable for system operation and dispatch.

Generally speaking, the challenges for diagram automatic generation of mega power grid are as follows: Firstly, there are large amount of equipments to be mapped into the automatically generated diagram. It is necessary to establish rules based on logical relationship to generate different levels of diagram for the power grid. Once the model of the power grid has changed, the new generated 
diagram should be able to inherit from previous version of diagram to realize incremental mapping, so as to reduce the difficulty and workload for maintenance of automatically generated diagram. the diagram automatic generation methods should be able to layout in horizontal and vertical style and effectively utilize the space on the diagram, so that the generated diagram is more consistent with the habits and requirements of system operators for system operation and simulation. Finally, for large scale grid power flow diagram, high display efficiency is particularly important and the viewport region of domain elements and their external connectivity has to be carefully calculated, so as to support huge amounts of equipments mapped in limited layout space. Dynamic viewport display technology is needed to support all kinds of browsing and operation on the generated diagrams.

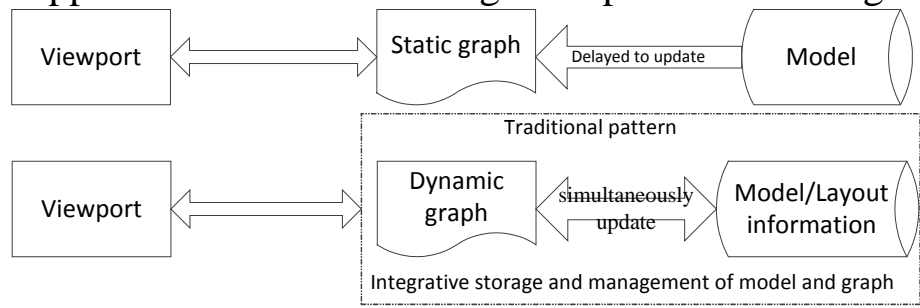

Fig. 1 Integrative management of model and graph

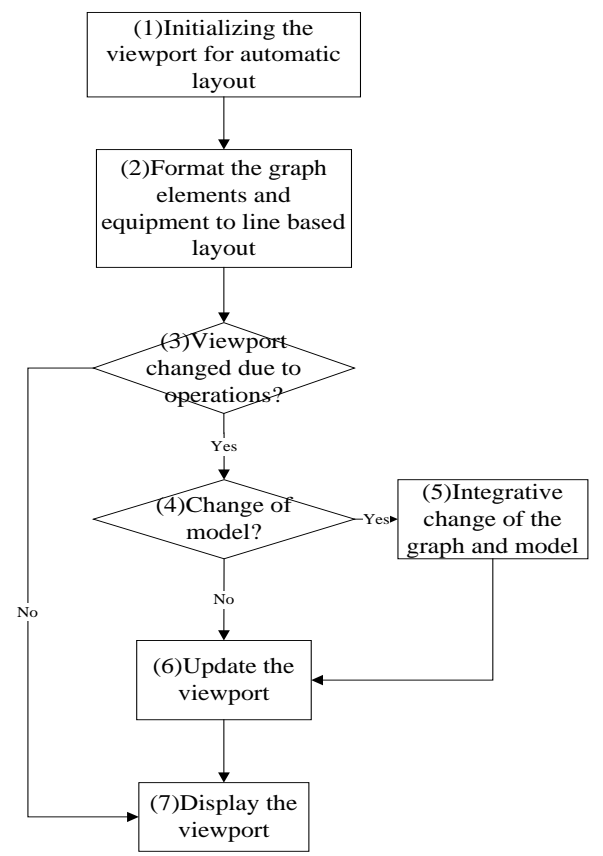

Fig. 2 Flowchart of line layout based dynamic viewport display method for automatic generation of power flow diagram

\section{Line layout based dynamic viewport display technology for diagram automatic generation}

Integrative management of model and graph. Traditionally, the model and graph of power grid is created and managed by regions, which means the modification and update of graph resource is relatively postponed than that of the model.

In our proposed method, we integrative manage the topology of the model and the automatic layout (graph). As shown in Fig.1, the equipments in the model are always consistent to the graphic elements in the generated diagrams. As benefits, it is possible to quickly search between model and graph of the power grid in the region of the viewport and support incremental update of the diagrams.

Dynamic viewport display method for automatic generation of power flow diagram. Based on integrative management of model and graph, line layout based dynamic viewport display method is developed for power flow diagram automatic generation. The flowchart is show in Fig.2 and the demonstration diagram is show in Fig. 3. The process for automatic generation of power flow diagram is described as follow. 
(1) Initializing the viewport for automatic layout. At the beginning, we need initialize the display for layout of the power flow diagram. Generally, the left top point is selected as the zero point for the generated layout.

(2) Format the graph elements and equipments according to line based layout. In this step, the integrative file that contains both the model and graph is parsed and formatted into line based layout. Firstly, these graphic elements corresponding to physical objects in the model of power grid such as substations, transformers, buses are generated. Then these elements are arranged on different lines with different longitudinal $\mathrm{Y}$ axis migration according to their connectivity relationship. All the equipments in the model in the layout are managed by a matrix with line and column number. Then add the transmission lines and T connected lines between graphic elements according to the topology connectivity in the model.

(3) Viewport changed due to operations? Check whether the viewport has changed, if so, go to step (4), otherwise go to step (7).

(4) Change of model? Check whether the elements in the model have changed, if so, go to step (5), otherwise go to step (6).

(5) Integrative change of the graph and model. In dynamic viewport display, some operation may change the model of power grid and the graph need to be updated simultaneously. These operations include clipping or incremental loading of the model and graph. These operations are directly mapped into the equivalent operations of the both graph and model of power grid. With the update of both graph and model, the automatic generated layout is updated at the same time.

(6) Update the viewport. There are some operations on the generated diagram that need to update viewport. These operations include viewport display, display movement, equipment positioning, incremental display update, etc.

Once there are operations on the generated diagram, the viewport needs to be updated. Such operations include viewport displaying, display movement, equipment positioning, display incremental update, etc.

Display movement: when moving the display, the new viewport will be in horizontal and vertical directions with migration amount along both $\mathrm{X}$ and $\mathrm{Y}$ direction. The range of line and column numbers of elements on the layout is updated and recorded for the viewport according to the offsets between the new and old viewport.

Equipment positioning: get the line and column number of the equipment to be positioned and move the center of the viewport to the equipment. The range of line and column numbers is updated for displaying the layout in the new viewport.

Incremental display update: sum up the set of out of date elements within the scope of the viewport, delete the out of date elements and add the elements just in the new viewport. Then update and display the new viewport to accomplish incremental update of power flow diagram.

(7)Display the viewport. Set the initial viewport as the area from left and top point to the actual physical display point of the view window; then calculate the region of effective visible graph and corresponding equipments in the model and the visual range of line and column numbers. Display the visual region of the viewport. Whenever the viewport is changed, the new region is recalculated and range of line and column numbers that record the region of viewport are updated.

\section{Case study}

Fig. 4 is one of the generated power flow diagrams for large scale power grid. In the diagram, some voltage level of a substation is of interest to the system operators and such bus bars are expended to show the details, while other substations are shown without details as rectangle boxes. On the generated diagram, the measurements are automatically mapped, and with dynamic viewport display technology, we can filter, clip, or incremental update of the graphic elements with high efficiency. Besides, advanced analysis functions can be applied on the generated diagram, such as power flow calculation, state estimation, etc. 


\section{Summary}

In this paper, we propose dynamic viewport display method for automatic generation of power flow diagram for large scale power grid.

The method for integrative management of model and graph is introduced to guarantee the consistency between the model and graph of the grid and the incremental update of generated diagram. Dynamic viewport display method is developed to automatically generate of the power flow diagram for large scale power grid and support efficient graphical operations.

The proposed method is applicable and easy accomplished to realize automatic generation of power flow diagram for mega power grid. The example in actual projects has shown its advantages.

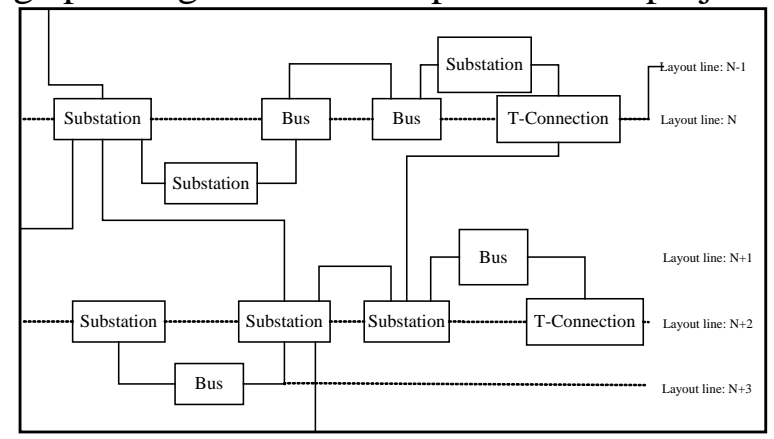

Fig. 3 Demonstration diagram based on line layout based dynamic viewport method

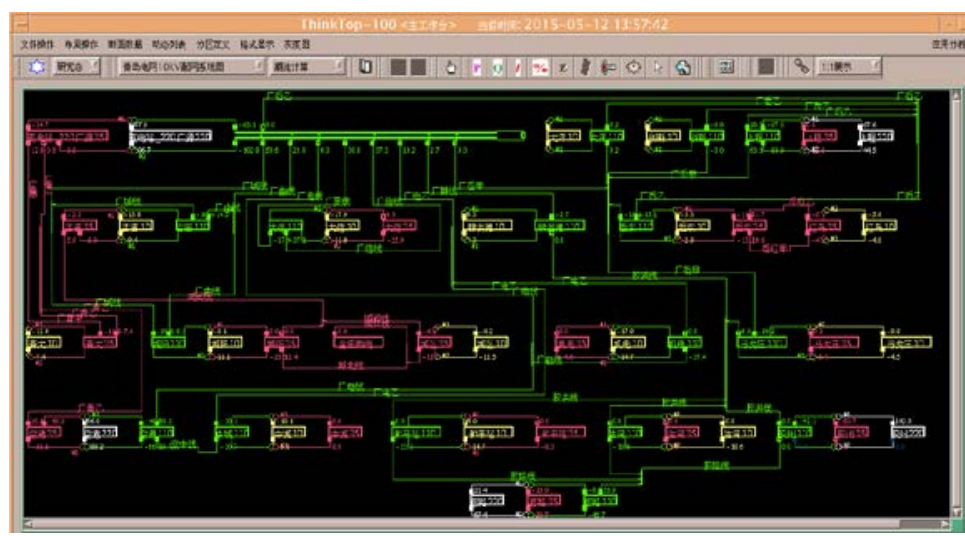

Fig. 4 Automatically generated power flow diagram in actual project

\section{Acknowledgments}

This work is sponsored by China Southern Power Grid (CSG) Technology Program (No. K-ZD2013-006.)

\section{References}

[1] Protsko, L.B., Sorenson, P.G., Tremblay, J. P., Schaefer, D.A. IEEE Trans. Software Eng. Vol.17 (1991) 10-21.

[2] Rochefort, M., DeGuise, N., Gingras, L. Elect. Power Syst. Res. Vol.36 (1996), 203-210.

[3] Ong, Y.S., Gooi, H.B., Chan, C.K. IEEE Proceedings Generation. Transmission and Distribution, Vol.147 (2000), 292-298.

[4] SONG S.Y., HE G.Y, XU P.L. and et al. Automation of Electric Power Systems, Vol. 33(2007), 12-15.

[5] ZHANG J.M., WANG Y., GU W. Automation of Electric Power Systems, Vol. 33(2009), 43-47. 
[6] ZHANG J.M., YE Y., CHEN L.Y. and et al. Automation of Electric Power Systems, Vol. 37 (2013), 107-112 